\title{
A Proposed Expert System for Nursing Practice
}

\author{
A Springboard to Nursing Science
}

\author{
Judy G. Ozbolt, Samuel Schultz II, Mary Ann Swain, and \\ Ivo L. Abraham
}

\begin{abstract}
The knowledge on which nursing practice is based comes largely from traditional sources, expert nurses passing on the wisdom of their experience to novices. Nursing research, although increasing, is usually parallel to nursing practice, and its findings, at best, are implemented only after long delays. Consequently, the most effective nursing responses to a particular client problem may be undiscovered or unknown. Nursing information systems reflect the nature and usage of nursing knowledge. They offer standard care plans, but the knowledge and decision structures for individualizing care remain exclusively in the mind of the nurse. Nurses may have great freedom to enter information into the information system, but the information is rarely retrievable in a form suitable for evaluation or research. Nursing practice, and the knowledge on which it is based, could be enhanced through the use of a novel expert system. This paper describes how such a system could be developed, with examples from the authors' prototype programs. Taxonomies of data, diagnoses, objectives, and interventions would make it possible to compare patients and to determine the relative effectiveness of nursing interventions. A builtin evaluation component would provide feedback and correction. Everyday nursing practice would become a field for research, and the knowledge gained from research would immediately be fed back into practice. In its development and in its implementation, this kind of system would help to build nursing science.
\end{abstract}

\section{INTRODUCTION}

For more than a century, nurses have been developing and communicating the knowledge on which their practice is based. Significant scientific research on nursing practice, however, has been under way only since the 1960s. Most nursing knowledge, therefore, has been gleaned from the clinical experience of practitioners and passed down from experts to novices. As a result, it is often idiosyncratic in conceptualization and untested

From the Center for Nursing Research, The University of Michigan, Ann Arbor, Michigan 48109, and the School of Nursing, Case Western Reserve University, Cleveland, Ohio 44106. 
in validity and reliability. Early information systems for nursing care have reflected the largely unsystematic nature of nursing knowledge. That is, they have allowed nurses to share and communicate the kinds of information they believe are useful, but they have provided only the most rudimentary decision support-i.e., standard care plans associated with common problems. Decisions about how to derive diagnoses from data and how to individualize the care plan rest with the cognitive and intuitive processes of the nurse. Developing the next generation of nursing information systems will require that nursing knowledge be codified for incorporation into the systems. Once such systems are in place, they will provide a mechanism for systematically testing existing knowledge and feeding back new knowledge into practice.

\section{THE NATURE OF NURSING KNOWLEDGE}

\section{What Kind of Knowledge Does Nursing Need?}

Before Florence Nightingale, nursing was assumed to consist of the intuitive, nurturant action "natural" to any woman. Nightingale 1 established that nursing was the application of specialized knowledge and skills, and that the knowledge and skills had to be learned. Nightingale saw nursing as "the proper use of fresh air, light, warmth, cleanliness, quiet, and the proper selection and administration of diet-all at the least expense of vital power to the patient."1 In her view, then, nurses required knowledge of basic principles of hygiene, comfort, and energy conservation, and skill in implementing them.

Since Nightingale's time nursing has become increasingly complex and sophisticated. Although Nightingale's principles have not been abandoned, many nurses have attempted to give a more comprehensive description of nursing as its is practiced today. One of the more influential descriptions, that of Virginia Henderson, ${ }^{2}$ has been adopted by the International Council of Nurses and by many other nursing organizations, schools, and agencies worldwide:

\footnotetext{
Nursing is primarily assisting the individual (sick or well) in the performance of those activities contributing to health, or its recovery (or to a peaceful death) that he would perform unaided if he had the necessary strength, will, or knowledge. It is likewise the unique contribution of nursing to help the individual to be independent of such assistance as soon as possible. ${ }^{2}$
}

According to Henderson, nurses need to know which activities will contribute to health or to its recovery or to a peaceful death. They must be skilled in performing these activities and in helping others to become able to perform them. All this implies that nurses are able to distinguish between health and illness, to set appropriate goals, to select those actions most likely to help the individual, and to foster the strength, will, and knowledge that promote independence.

In the 1960s and 1970s many nurses developed descriptions of their concepts of nursing. Orem' $\mathrm{s}^{3,4}$ description of nursing as the provision and management of self-care seems to be a direct descendant of Henderson's, ${ }^{2}$ and so would require similar kinds of knowledge. Roy, ${ }^{5,6}$ on the other hand, said that the purpose of nursing was to promote adaptation. Nurses practicing within this framework would need to know how to rec- 
ognize positive adaptation and maladaptation and how to identify and modify the stimuli impinging on a patient. Rogers ${ }^{7}$ offered yet another perspective, saying that the focus of nursing is "unitary man," an indivisible whole that is greater than the sum of its parts and that is in continuous interaction with the universe. According to Rogers, nurses must interact with the whole person rather than focusing on parts of the person, but she offered little guidance about how to do this or with what objectives, or about the kinds of knowledge that would be needed.

More recently, Erickson, Tomlin, and Swain ${ }^{8}$ have developed a theory and paradigm of nursing that, while original in perspective, incorporates many elements of earlier descriptions of nursing. According to their definition,

Nursing is the holistic helping of persons with their self-care activities in relation to health. This is an interactive, interpersonal process that nurtures strengths to enable development, release, and channeling of resources for coping with one's circumstances and environment. The goal is to achieve a state of perceived optimum health and contentment. ${ }^{8}$

Nurses who use this paradigm would require knowledge of healthy functioning and human development as well as the clinical sensitivity and interpersonal skills to identify each client's strengths and needs and to select appropriate responses to promote health.

All these definitions depict nursing as nurturant, as focusing on care rather than cure. Although there is considerable overlap among conceptual frameworks in the content of the knowledge needed for nursing practice, there are differences in perspective, emphasis, and terminology. Nurses have attempted to apply knowledge derived from practice or borrowed from other disciplines to one or another of these frameworks. Only recently, however, have some researchers begun to develop knowledge within particular nursing frameworks, describing nursing phenomena from a consistent perspective and empirically defining relationships among them. The lack of a well-developed epistemological system of nursing knowledge hinders nursing practice and inhibits the development of computer systems to facilitate nursing's cognitive tasks. Progress in nursing would be advanced by programs of research within selected frameworks to develop coherent bodies of knowledge.

\section{What Kinds of Knowledge Does Nursing Have?}

Although there is great diversity in the conceptual models of nursing, most nurses agree that they provide care via a problem-solving process that includes collecting and analyzing data, formulating diagnoses, setting objectives, choosing and implementing interventions, and evaluating outcomes. This process is depicted in Figure 1.

The general model of the nursing process is shown as an open system with a feedback loop. When interventions have been implemented, this information is fed back into the system. New data are collected and new diagnoses are synthesized. These new diagnoses are, in effect, outcomes of the care that has already been given. They are compared with the original diagnoses and the objectives to determine whether the client has progressed, regressed, or stayed the same. The next step is to analyze the causes of success or failure. If the intervention was effective in helping the client to achieve the objective or to move toward the objective, this is noted for future reference. If the client did not achieve the objective, the nurse must ask why. Was the diagnosis incorrect? Was the objective inappropriate or unrealistic? Was the intervention ineffective? Was the target date too 


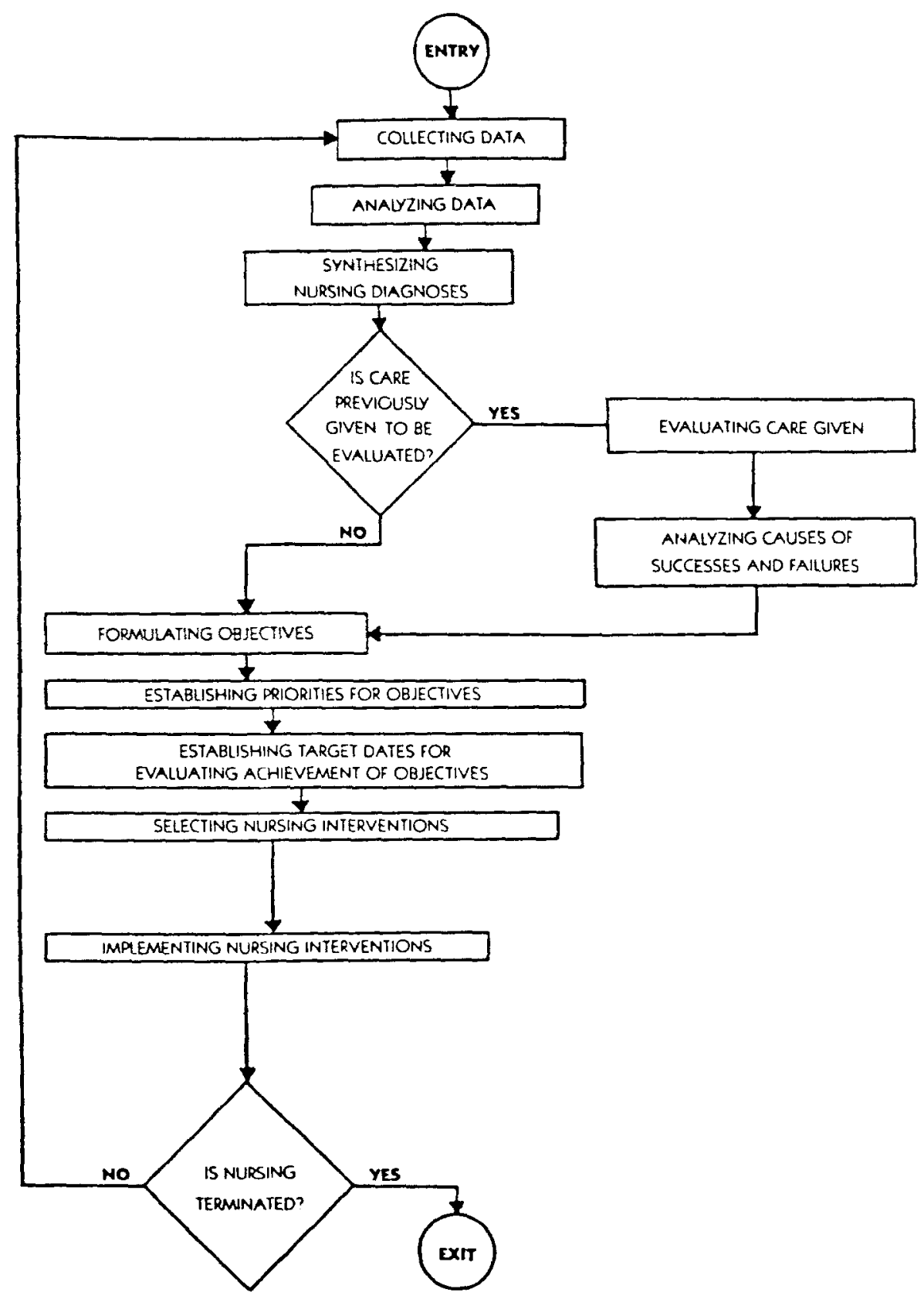

Figure. 1. General model of the nursing process.

early? The nurse investigates, makes a judgment, and continues the process, setting new objectives and target dates, choosing and implementing new interventions (or continuing with the same ones), and evaluating the results. This goes on, ideally, until the diagnoses indicate that the client has no problems requiring nursing assistance. The nurse and client 
choose objectives and interventions related to termination of the professional relationship and exit from the system.

Although this model is a simplified and idealized representation of the complexities of nursing practice, the process it depicts is generally accepted in professional circies as the means by which nurses ought to deliver care. Indeed, the American Nurses's Association ${ }^{9}$ has set standards for each step of the nursing process. Yet nurses often have difficulty implementing the process, even where information systems have been set up to support the process. ${ }^{10}$

One source of difficulty is the fragmentary nature of knowledge at each step of the process. Note that in the general model shown in Figure 1, the content of the boxes is not defined. What data are to be collected? What are possible diagnoses, objectives, and interventions? Nurses have used each of four general "methods of knowing" 11 to answer these questions. These methods are tenacity, authority, intuition, and science

In the method of tenacity people hold firmly to what they know to be true because they have always known it to be true. In the nursing process the method of tenacity results in ritualistic action. Nurses collect certain data because they believe they must have the data because they have always collected them, even if they don't know what to do with the data once collected. The method of tenacity can also result in ritualistic interventions, always treating a problem in the same way because "that's the way it's done here." When nurses operationalize the nursing process using knowledge derived from the method of tenacity, they risk perpetuating false knowledge and overlooking valid but less traditional knowledge.

Nurses who use the method of authority accept certain knowledge because it is declared to be true by a recognized authority or authorities. Nurses are using the method of authority when they choose particular interventions because a nursing textbook recommends them, or when they carry out a treatment according to the instructions in a procedure manual. The method of authority is a useful way of knowing since it would be impossible for every nurse personally to conduct empirical tests of all knowledge before accepting it. Like the method of tenacity, however, the method of authority may result in accepting invalid knowledge and ignoring valid knowledge.

The third method of knowing, the a priori method or the method of intuition, relies on common sense to determine what is true. Nurses use the a priori method when they review a mass of patient data and make an intellectual leap to diagnoses, or when they consider alternative objectives and interventions and, on the basis of careful reasoning (but no scientific data), select those they believe to be most appropriate for their clients. The hazard in this method is that reasonable people can disagree, and the a priori method itself cannot resolve the dispute by determining who is correct.

Finally, nurses are increasingly using the method of science. This method of knowing is characterized by objectivity, as propositions are put to empirical tests, and is capable of self-correction on the basis of the results of those tests. Particularly since the 1960s, an increasing number of studies have described nursing phenomena and relationships and provided evidence of the relative effectiveness of nursing interventions. Because nurse researchers conduct their investigations from a variety of perspectives on the nature of nursing or from no explicit nursing perspective at all, however, it is difficult or impossible to synthesize their findings into a coherently growing body of knowledge. How, for example, does one relate one researcher's findings about how to promote 
adaptation to another researcher's description of self-care assets and deficits? And how can these diverse bits of knowledge be used to operationalize nursing data, diagnoses, objectives, and interventions? Such difficulties have hindered the implementation of research knowledge in nursing practice. The problem is compounded by the fact that most researchers are not practicing clinicians, and most nurses are not researchers; in 1978 only $5 \%$ of nurses held a master's or doctoral degree. ${ }^{12}$ Consequently, research often goes on parallel to the world of nursing practice, and findings are implemented after long delay, if at all.

If nursing knowledge is an amalgam of ritual, tradition, intuition, and science, how is it organized and used? In some settings, nurses have identified nursing problems that occur frequently on their units and have devised standard care plans for those problems. A variety of classification schemes for patient problems have been developed and implemented in hospitals and other health care agencies. In reviewing some of these schemes and considering the North American Nursing Diagnosis Association's efforts to classify its own set of diagnoses, Kritek $^{13}$ proposed that nurses need to differentiate between data and diagnoses, which are confounded in many attempts at classification; to find ways of classifying that are consistent with holistic nursing, rather than splitting persons into minds and bodies; to identify the common denominators in existing classification schemes and assess what is useful; and to find organizing principles appropriate to nursing.

If nurses need to find useful and appropriate ways to classify nursing data and diagnoses, the same is true for nursing objectives and interventions. Meanwhile, for most nurses in most situations, the organization of nursing knowledge is personal and idiosyncratic. Each nurse decides, on the basis of his or her own synthesis of knowledge, the data to collect, the problems to attack, the objectives to pursue, and the interventions to implement. One nurse's experience remains highly individualized and difficult to integrate with another's. Thus, although nurses have learned a great deal about practice from experience and from research, nursing knowledge is disunified and fragmentary. It will remain so until nurses find organizing principles to permit its systematic testing, synthesis, and codification.

\section{EXISTING NURSING INFORMATION SYSTEMS}

\section{Representative Systems}

The first comprehensive hospital information system was the Technicon system at El Camino Hospital in Mountain View, California. Its nursing component reflects the state of nursing knowledge at the time of its development, the late 1960s and early 1970s. Charged with devising a way to computerize nursing notes, the traditional narrative charting of patient conditions and nurse actions, a committee of nurses recognized that their task was a more fundamental one, to develop a comprehensive, integrated system for processing nursing care data, a system that would include care planning, charting, and feedback for audit. ${ }^{14}$ Relying primarily on the methods of authority and intuition, they identified common nursing problems frequently associated with certain medical diagnoses and developed standard care plans for the nursing problems. ${ }^{15}$ Ultimately, a menu-driven system was developed wherein nurses use a light pen and a keyboard to 
enter patient data, select and modify nursing problems and care plans, and record nursing actions and patient progress.

For example, a medical diagnosis of cholecystitis or cholelithiasis will cause the system to produce a nursing care plan that includes four "usual problems": pain, nausea, potential abdominal distention, and potential misunderstanding of diet at home in relation to diagnosis. For each problem, the system also produces a list of expected outcomes, deadlines, and nursing orders. ${ }^{15}$ This standard care plan will be produced for any patient with a medial diagnosis of cholecystitis or cholelithiasis. The nurse can then modify the care plan or add "unusual problems" and related outcornes, deadlines, and nursing orders. Note that the system itself produces a standard care plan in response to the cue of a medical diagnosis. It is not constructed to compare patient data to a nursing knowledge base and reach decisions about nursing problems or nursing care. Those activities must be done by the nurse.

This fact allows nurses in different settings using the Technicon system to organize their thinking about nursing in their own ways. In the Technicon installation at the NIH Clinical Center, for example, Mayers's ${ }^{16}$ common nursing problems and standard care plans are not used. Instead, the nursing process has been operationalized in a framework based on Maslow's ${ }^{17}$ hierarchy of needs, with a different set of standard care plans. Still, it is up to the nurse, not the computer system, to classify data according to need categories, to determine the nursing problems, and to select and modify care plans appropriately. ${ }^{10}$

Although developed later, the nursing care plan module of IBM's Patient Care system bears significant similarities to the nursing component of the Technicon system. The system stores a limited number of nursing diagnoses (one hospital reports having 21 nursing diagnoses on file) with associated care plans. It is up to the nurse to decide which if any of the stored nursing diagnoses applies to a particular patient. The nurse may then call up the relevant diagnoses and care plans and edit the care plans to correspond to the patient's situation. Components of the diagnosis and care plan include the following: (1) statement of the diagnosis (e.g., "decreased mobility/immobility"); (2) identifying information about the patient (age, sex, room number, physician); (3) nursing measures - assessments (what to assess, e.g., mobility, capabilities, weakness); (4) nursing measures - interventions (what to do, e.g., mobilize by hall walk with crutches Q.I.D.); (5) nursing measures-teaching (what to teach, e.g., appropriate activity and rest); (6) goals (e.g., maximum mobility, ambulates independently); (7) evaluation (whether or not goals are met, whether care plan is to be continued, revised, or discontinued). ${ }^{18}$

It is apparent that the diagnostic statements are quite broad, and the care plans are very general. These features permit a small number of diagnoses and care plans to apply to a large number of patients. The question might be raised as to whether such broad and general care plans are useful if the goal is to provide individualized care. The computer, however, provides no assistance in individualizing the care plan. If the care plan is to be individualized, the nurse must do it.

\section{Nursing Knowledge and Existing Systems}

The Technicon and IBM systems, like other commercially available nursing information systems, reflect many of the limitations in nursing knowledge described earlier. 
They are adaptable to many different conceptualizations of nursing, or may be used with no explicit or consistent conceptualization at all. The nursing knowledge needed to produce standard problem lists and care plans may be derived from tenacity, authority, intuition, or research, but once placed in the system it remains static. The more complex and fluid knowledge and decision structures necessary to design and evaluate care appropriate to individual patients remains exclusively in the mind of the nurse. As repositories of standard care plans, existing nursing information systems are little more than filing cabinets for the most simplified forms of nursing knowledge. They neither capture the complexity and richness of nursing knowledge nor help to advance it.

\section{A PROPOSED EXPERT SYSTEM}

\section{The Knowledge Base}

It is possible to develop an expert system for nursing practice with a much richer and more complex knowledge base, one that can be used by the computer for artificially intelligent reasoning and one that will grow with experience. Such a knowledge base would require a high degree of internal consistency, since its elements and their relationships would have to be clearly and explicitly defined. How is this possible, given the "disunified and fragmentary" nature of nursing knowledge described previously? It can be achieved through a process of bootstrapping, starting from the knowledge that is currently available and strengthening and enriching it over time.

First, to achieve internal consistency in the knowledge base it is useful to select a conceptual model of nursing and to deduce taxonomies for each step of the nursing process. There is no compelling reason to choose one of nursing's conceptual models over another, but because of its practical emphasis on care and its own internal consistency our group has been working with Orem's. ${ }^{3,4}$ For future work we are considering a change to the Erickson, Tomlin, and Swain ${ }^{8}$ model because of its greater richness and complexity, but this paper will use examples from our work with Orem's model.

According to Orem, each person requires certain kinds of self-care to maintain or restore health or to promote normal development. When the person is unable to provide the necessary self-care because of illness, injury, or disability, or because the needed care requires specialized knowledge and skills, assistance in the form of nursing is called for. The nurse thus collects data to obtain evidence of the degree to which self-care requisites are met and the degree to which the client is able to meet them independently. $\mathrm{Orem}^{4}$ defined 14 categories of self-care requisites. These are as follows: (1) maintaining sufficient intakes of air, water, food; (2) providing care associated with eliminative processes and excrements; (3) maintaining a balance between activity and rest; (4) maintaining a balance between solitude and social interaction; (5) preventing hazards to life, functioning, and well-being; (6) promoting normalcy; (7) bringing about and maintaining living conditions that support life processes and promote the process of development; (8) providing care to prevent or mitigate the deleterious effects of conditions that can affect human development; (9) seeking and securing appropriate medical assistance when needed; (10) being aware of and attending to effects of pathological conditions or states; (11) effectively carrying out medically prescribed actions; (12) being aware of and attending to or regulating the discomforting or deleterious effects of medical care; (13) 
modifying the self-concept to accept one's state of health and need for specific forms of health care; (14) learning to live with the effects of illness, injury, and treatment in a way that promotes continued personal development. ${ }^{4}$

Specific data items can be identified in each category, and the relation of each item or combination of items to the status of self-care can be described. For example, items related to maintaining a sufficient intake of air include assessment for the presence of wheeze, stridor, and adventitious breath sounds. These items are related specifically to patency of airways. If the data tell us that airways are patent and if a number of other conditions are similarly satisfied, then we can conclude that the self-care requisite for maintaining a sufficient intake of air is met. For many categories of self-care requisites, valid and reliable measures for nursing assessment can be borrowed from the researchbased Horn-Swain ${ }^{19}$ instrument, which was also developed in the Orem framework. Other items will need to come from the literature or from nurses' experience or to be invented as a part of systems development.

Nursing diagnoses are easy to define within Orem's conceptual framework. Since the focus is on self-care, nursing diagnoses are statements of the degree to which selfcare requisites are met and of the degree to which the client is able to meet them independently. These statements are specific to the individual client and are based on the data collected. Because the relationships between data and diagnoses are clearly delineated, it is possible for the computer to derive diagnoses from the data. Thus, nurses using this sytem would no longer collect data to no purpose, or overlook important data and miss diagnoses.

The ability to generate client-specific diagnoses and care plans from the nursing assessment data has not been realized in any commercially available nursing information system. That this is possible, however, has been demonstrated by our prototype programs, ${ }^{20,21}$ which produce diagnoses like this:

Maintaining a sufficient intake of air is impaired by

a. infection or irritation in the lungs or airways

b. increased airflow resistance

Self-care agency for maintaining a sufficient intake of air is impaired by

a. inadequate knowledge related to alterations in respiratory function

appropriate actions to take when alterations occur

b. inability to adapt activity so that oxygen supply is equal to demand

c. embarrassment re clearing secretions in social situations

Nursing objectives are related to diagnoses and are statements to the effect that selfcare requisities will be met and that the ability to meet them independently will be maintained or augmented to the degree feasible. Like diagnoses, objectives are specific to the individual client. An example of objectives related to the diagnoses above would be:

Client will maintain a sufficient intake of air, with no further evidence of

a. infection or irritation in lungs of airways

b. increased airflow resistance 
Client's self-care agency for maintaining a sufficient intake of air will be augmented to include

a. adequate knowledge related to alterations in respiratory function appropriate actions to take when alterations occur

b. ability to adapt activity so that oxygen supply is equal to demand

c. resolution of embarrassment re clearing secretions in social situations.

Objectives are derived from the client data base, the diagnoses, and knowledge of what it is possible to achieve. Some of this knowledge is available from the research literature, but much of it would have to come from the opinions of expert nurses (the a priori method of knowing). Once an expert system was operational, however, it could aggregate data about the circumstances under which objectives were achieved and correct its own structures to propose more appropriate and achievable objectives.

Interventions, in this conceptual model, have to do with supplying the self-care the client is unable to do unassisted and promoting the client's self-care agency. They are also specific to the diagnoses and objectives. Since the research on effectiveness of nursing interventions does not begin to cover the number and variety of possible interventions as defined within this framework, the knowledge base for system development would be largely a priori. Again, however, evaluations of the effectiveness of interventions could be aggregated over time so that the system could learn from its own experience.

\section{The Decision Structures}

Once the elements of the knowledge base and their relationships have been defined, it is possible to develop decision mechanisms. Both the knowledge base and the client data base are extensive, and complex decisions must be made throughout the nursing process. Decision structures are needed that will produce accurate and reliable decisions without requiring inordinate time for data processing. Statistical decision models used in some systems for medical diagnosis are reliable but slow, overlooking associative processes in data acquisition and storage that could streamline search and retrieval. On the other hand, human inferential strategies, even as mimicked in some computerized decision-support systems, are subject to biases.

In an effort to overcome these limitations, we have proposed a multivariate mathematical approach to clinical decision making that permits the incorporation of information-processing strategies experts typically used while maintaining the statistical rigor of data-based systems. ${ }^{22,23}$ In this approach, both item information and associative information are used for clinical inference. Item information, the actual data that are acquired, is represented as a vector in which the features of the item constitute the elements. As each item is acquired, it is immediately integrated into a larger network of information. The integration of two items of information into a new item (mathematically, the convolution of two item vectors into a composite vector) results in associative information. The clinical condition of a patient at any time is therefore defined as a vector composed of both item and associative information acquired to that time, with appropriate weighting and with a defocusing factor indicating loss of information (since information acquired earlier may be partially lost by the time of a subsequent assessment). Because 
clinical inference includes not only a description of the patient's condition at any time, but also a judgment of the normality or abnormality of the condition, the clinical condition vector is next compared to upper- and lower-limit vectors representing the population criterion interval. These population criteria are stored in a normative data base, or knowledge base. If the patient condition vector falls outside these limits, the condition is considered abnormal. The proposed decision structures thus combine the associative processes of data acquisition and storage generally found in heuristic approaches with the normative comparisons of normality and abnormality commonly found in statistical approaches. Although we have not yet tested this approach in a prototype program, we are hopeful that it will provide an effective and efficient tool for clinical decision support in nursing.

\section{Toward the Development of Nursing Science}

The creation and implementation of an expert system such as the one described here would do much to advance the development of nursing science. Work already completed on prototype programs demonstrates the feasibility of organizing and codifying nursing knowledge, even when some of the knowiedge is based on tradition, experience, or common sense rather than research. A system so organized and codified, with defined and traceable decision structures, would be an invaluable resourse for research and evaluation. A taxonomy of patient data with valid and reliable measures combined with a taxonomy of nursing diagnoses reliably derived from the data would make it possible to compare patients to determine similarities and differences in their problems, strengths, and responses. Taxonomies of objectives and interventions would make it possible to determine the relative effectiveness of interventions in achieving the objectives, given particular patterns of nursing diagnoses. A built-in evaluation component that took note of successes and failures and accordingly adjusted the system's decision making would provide quick feedback and correction. The day-to-day activities of nursing practice would automatically and continuously be the subject of research, as the sytem aggregated data and interpreted successes and failures. Furthermore, the knowledge from that research would be immediately fed back into practice.

An expert system, then, would contribute to nursing knowledge first by requiring that existing knowledge be organized and codified for the development of the system. It would make a still greater contribution by making the everyday practice of nursing amenable to research and by putting back into practice the knowledge gained from that research. The development of expert systems for nursing need not await fuller emergence of nursing science; on the contrary, expert systems can become a springboard to nursing science.

\section{REFERENCES}

1. Nightingale, F., Notes on nursing: What it is and what it is not, Appleton, New York, 1860. Republished Dover, New York, 1969.

2. Henderson, V., ICN basic principles of nursing care, ICN (International Council of Nurses) House, London, 1960. 
3. Orem, D. E., Nursing: Concepts of practice, McGraw-Hill, New York, 1971.

4. Orem, D. E., Nursing: Concepts of practice, 2nd ed., McGraw-Hill, New York, 1980.

5. Roy, C., Sr., Adaptation: A conceptual framework for nursing. Nursing Outlook 18(3):42-45, 1970.

6. Roy, C., Sr., Adaptation: A basis for nursing practice. Nursing Outlook 19:254-257, 1971.

7. Rogers, M. E., An introduction to the theoretical basis of nursing, Davis, Philadelphia, 1970.

8. Erickson, H. C., Tomlin, E. M., and Swain, M. A. P., Modeling and role-modeling; a theory and paradigm for nursing, Prentice-Hall, Englewood Cliffs, New Jersey, 1983.

9. American Nurse's Association, Nursing: A social policy statement. Author, Kansas City, Missouri, 1980.

10. Romano, C., Documentation of nursing practice using a computerized medical information system. Proceedings of the fifth annual symposium on computer applications in medical care, IEEE Computer Society, Los Angeles, 1981.

11. Kerlinger, F. N., Foundations of behavioral research, 2nd ed., Holt, Rinehart \& Winston, New York, 1973.

12. Moses, E. A., and Roth, A., Nursepower: What do statistics reveal about the nation's nurses. Am. J. Nursing 79:1745-1756, 1979.

13. Kritek, P. B., Current nomenclature and classification systems. Classification of nursing diagnoses: Proceedings of the fifth national conference (M. J. Kim, G. K. McFarland, and A. M. McLane, eds.), Mosby, St. Louis, 1984.

14. Gall, J. E., Demonstration and evaluation of a total hospital information system (DHEW Pub. No. (HRA) 77-31880), U.S. Government Printing Office, Washington, D.C., 1977.

15. Mayers, M. G., and El Camino Hospital, Standard nursing care plans, Vol. 1, K.P. Medical Systems, Palo Alto, 1974.

16. Mayers, M. G., A systematic approach to the nursing care plan, Appleton-Century-Crofts, New York, 1972.

17. Maslow, A., Toward a psychology of being, Van Nostrand, Princeton, 1962.

18. Light, N., Computers in nursing practice: On-line nursing care plans. Comput. Nursing 1(3):4, 1983.

19. Horn, B. J., and Swain, M. A., Criterion measures of nursing care quality: A health status instrument (PHS Publ. No. 78-3187), U.S. Government Printing Office, Washington, D.C., 1978.

20. Ozbolt, J. G., A prototype information system to aid nursing decisions. Proceedings of the sixth annual symposium on computer applications in medical care, IEEE Computer Society, Silver Spring, Maryland, 1982.

21. Ozbolt, J. G., Schultz II, S., Swain, M. A. P., Abraham, I. L., and Stein, K. F., Developing an expert system for nursing practice. Proceedings of the eighth annual symposium on computer applications in medical care, IEEE Computer Society, Siver Spring, Maryland, 1984.

22. Abraham, I. L., Schultz II, S., Ozbolt, J. G., and Swain, M. A. P., A multivariate algorithm for diagnostic information systems: Data acquisition and storage procedures. Proceedings of the Fifth Congress of the European Federation for Medical Informatics, Belgian Society for Medican Informatics, Brussels, 1984.

23. Abraham, I. L., Schultz II, S., Ozbolt, J. G., and Swain, M. A. P., A mutivariate mathematical algorithm for diagnostic information systems: Procedures for clinical inference. Proceedings of the Eighth Annual Symposium on Computer Applications in Medical Care, IEEE Computer Society, Silver Spring, Maryland, 1984. 